\title{
Monte-Carlo Based On-Orbit Single Event Upset Rate Prediction for a Radiation Hardened by Design Latch
}

\author{
Kevin M. Warren, Brian D. Sierawski, Robert A. Reed, Robert A. Weller, Carl Carmichael, Austin Lesea, \\ Marcus H. Mendenhall, Paul E. Dodd, Ron D. Schrimpf, Lloyd W. Massengill, Tan Hoang, Hsing Wan, \\ J. L. De Jong, Rick Padovani, and Joe J. Fabula
}

\begin{abstract}
Heavy ion cross section data taken from a hardened-by-design circuit are presented which deviate from the traditional single sensitive volume or classical rectangular parallelepiped model of single event upset. TCAD and SPICE analysis demonstrate a SEU mechanism dominated by multiple node charge collection. Monte Carlo simulation is used to model the response and predict an on-orbit error rate.
\end{abstract}

Index Terms-Geant4, MRED, rate prediction, SEU.

\section{INTRODUCTION}

I $\mathrm{N}$ space applications requiring the maximum possible single event upset (SEU) immunity at the level of the single storage element, it is common practice to design devices that are immune to single-node charge collection mechanisms. However, with reduction in feature sizes, single-node hardening techniques can be undermined by multiple-node charge collection processes [1]. In such cases, quantifying the on-orbit soft error rates (SER) can be difficult or impossible using SER prediction models developed for single sensitive volume upset mechanisms [2], since SEU result from coincident charge generation or collection in two or more transistors.

Monte Carlo, physics based simulations allow for greater flexibility in the definition and monitoring of charge deposition in single or multiple sensitive volumes and have found use in evaluating single event upsets in both terrestrial and space

Manuscript received July 20, 2007; revised August 23, 2007. This work was supported in part by Rad Hard Electronics for Space Exploration (RHESE) and NASA Electronic Parts and Packaging (NEPP). The portion of this work performed at Sandia was supported by the U. S. Department of Energy. Sandia is a multiprogram laboratory operated by Sandia Corporation, a Lockheed Martin Company, for the U.S. Department of Energy's National Nuclear Security Administration under contract DE-AC04-94AL85000. The computational portion of this work was supported by Vanderbilt University's Advanced Computing Center for Research and Education (ACCRE).

K. M. Warren and B. D. Sierawski are with the Institute for Space and Defense Electronics, Vanderbilt University, Nashville, TN 37203 USA (e-mail: kevin.m. warren@vanderbilt.edu).

R. A. Reed, R. A. Weller, R. D. Schrimpf, and L. W. Massengill are with the Electrical Engineering and Computer Science Department, Vanderbilt University, Nashville, TN 37235 USA.

C. Carmichael, A. Lesea, T. Hoang, H. Wan, J. L. De Jong, R. Padovani, and J. J. Fabula are with Xilinx Inc., San Jose, CA 95124 USA.

M. H. Mendenhall is with the Vanderbilt University Free Electron Laser Facility, Nashville, TN 37235 USA.

P. E. Dodd is with Sandia National Laboratories, Albuquerque, NM 87185 USA.

Digital Object Identifier 10.1109/TNS.2007.907678 applications [3]-[6]. However, high flexibility often comes at the price of programming complexity and simulation time.

To assist in the rapid development of SEU models and soft error rate prediction for specific applications in microelectronics, we have developed a software tool called MRED (Monte Carlo Radiative Energy Deposition). MRED is a Geant4 [7] application that includes a custom Python application-programming interface (API) for rapid reconfiguration and real-time analysis of events. Python is a dynamic object-oriented programming language, which offers strong support for integration with other languages and tools [8]. This software configuration preserves the underlying speed of the simulation while fully integrating a high-level programming language with components specifically tailored to radiation effects.

In this paper, the SEU response of a hardened-by-design latch, fabricated in a $90 \mathrm{~nm}$ technology, is analyzed. Angular dependencies on the measured heavy ion SEU cross section along both axes were determined to be results of multiple-node charge collection processes. A novel multiple volume, multiple transistor energy tracking method is developed in MRED, calibrated to heavy ion data, and shown to reflect heavy ion SEU cross sections. The ability of the model to reproduce the complex features of the experimental data and its subsequent application to on-orbit SER prediction represents a significant advancement in error-rate prediction techniques.

\section{LATCH DESIGN}

The latch circuit design is that of a dual interlocked cell and a detailed discussion of its operation is contained in [9]. Ion strikes to a single node will not result in a SEU. However, the state can be corrupted if a single particle deposits sufficient energy to simultaneously perturb the potential of two sensitive nodes [10].

The 12-transistor SEU hardened latch is fabricated on a 90 nm CMOS epitaxial, 9 layer metal process. Schematic and annotated layout views of the circuit are shown in Fig. 1. Further SEU resistance was achieved through spatial separation of the NMOS transistors of the redundant storage elements (e.g., NO and N2).

\section{EXPERIMENTAL}

Heavy ion irradiations were performed using $10 \mathrm{MeV} / \mathrm{u}$ $\mathrm{Xe}\left(58.7 \mathrm{MeVcm}^{2} / \mathrm{mg}\right), \mathrm{Kr}\left(31.2 \mathrm{MeVcm}^{2} / \mathrm{mg}\right)$, and $\mathrm{Ar}(9.7$ $\mathrm{MeVcm}^{2} / \mathrm{mg}$ ) beams. Static SEU cross sections are shown 


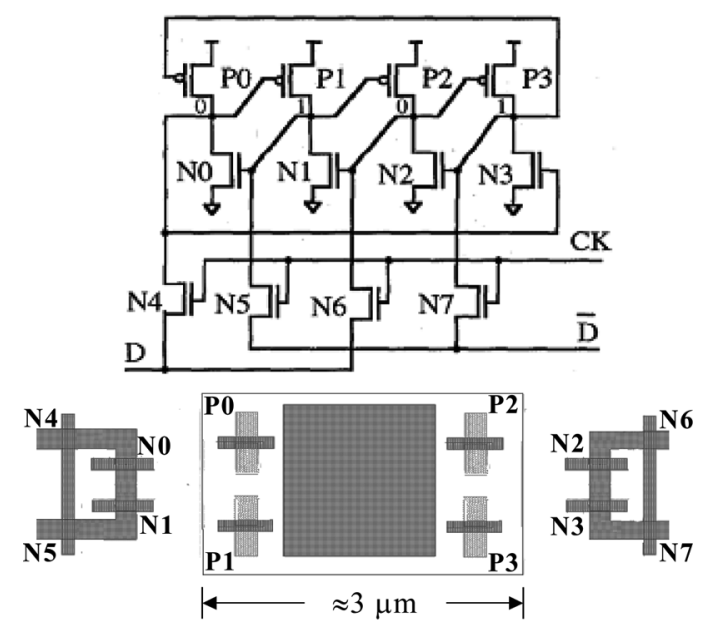

Fig. 1. Schematic and annotated layout views of the latch. Spatial separation of sensitive node pairs reduces the probability of multiple-node effects such as charge sharing, especially between NMOS transistors.

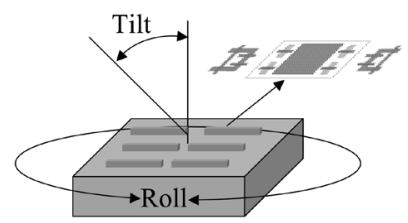

Fig. 2. Orientation of the ion beam with respect to the DUT surface and related nomenclature. A tilt angle of zero degrees refers to a normally incident beam. A zero degree roll angle refers to a beam direction along the long axis of the latch, also shown in Fig. 1.

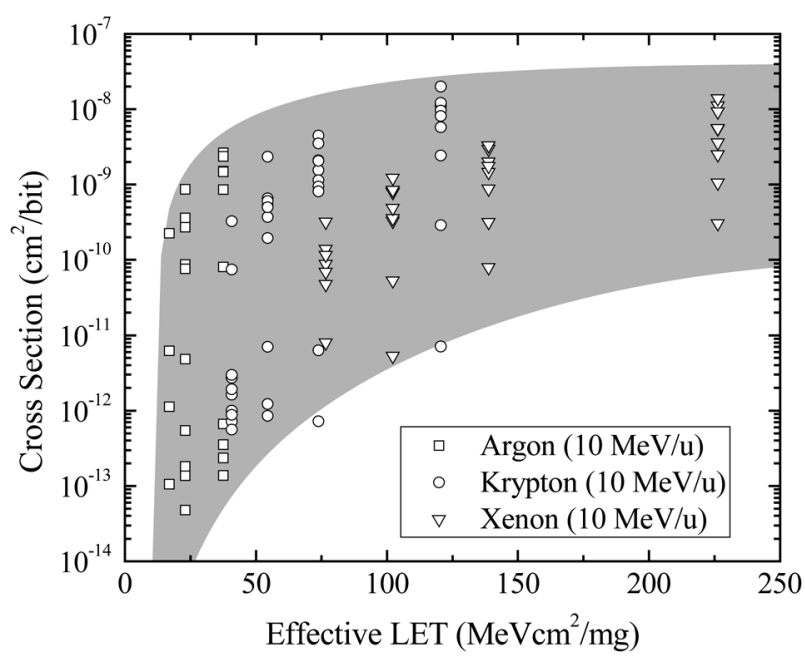

Fig. 3. Compilation of heavy ion data from irradiation of the latch shown in the classical effective LET manner. The span of SEU cross section for a given LET is due to the dependence of cross section on roll angle.

in Fig. 3 at tilt angles between 0 and 75 degrees and over a 180-degree range of roll angles. In this discussion, tilt refers to the angle between the normal to the device under test (DUT, Fig. 1) surface plane and the beam direction. Roll refers to the relative position of the DUT about the beam direction (Fig. 2). The devices were programmed in a checkerboard pattern (spatially alternating 1 's and 0 's).

The SEU cross section is presented in Fig. 3 as a function of effective Linear Energy Transfer (LET) in the customary
TABLE I

Latch Sensitive Node Pairs and CRitical Charges

\begin{tabular}{|l|c|c|c|c|}
\hline \multicolumn{5}{|c|}{$T^{|c|}$ Pair $Q_{\text {crit }}$ (fC) and Data State } \\
\hline & \multicolumn{2}{|c|}{$\mathrm{D}=0$} & \multicolumn{2}{c|}{$\mathrm{D}=1$} \\
\hline & $\mathrm{Q}_{\text {crit }} \mathrm{Tx}(\mathrm{A})$ & $\mathrm{Q}_{\text {crit }} \mathrm{Tx}(\mathrm{B})$ & $\mathrm{Q}_{\text {crit }} \mathrm{Tx}(\mathrm{A})$ & $\mathrm{Q}_{\text {crit }} \mathrm{Tx}(\mathrm{B})$ \\
\hline $\mathrm{N} 0 / \mathrm{N} 2$ & 6.3 & 2.5 & $\mathrm{~N} / \mathrm{A}$ & $\mathrm{N} / \mathrm{A}$ \\
\hline $\mathrm{P} 1 / \mathrm{P} 3$ & 13.9 & 2.5 & $\mathrm{~N} / \mathrm{A}$ & $\mathrm{N} / \mathrm{A}$ \\
\hline $\mathrm{N} 0 / \mathrm{P} 1$ & 4.4 & 1.7 & $\mathrm{~N} / \mathrm{A}$ & $\mathrm{N} / \mathrm{A}$ \\
\hline $\mathrm{N} 2 / \mathrm{P} 3$ & 3.2 & 1.8 & $\mathrm{~N} / \mathrm{A}$ & $\mathrm{N} / \mathrm{A}$ \\
\hline $\mathrm{N} 2 / \mathrm{N} 0$ & 2.5 & 6.3 & $\mathrm{~N} / \mathrm{A}$ & $\mathrm{N} / \mathrm{A}$ \\
\hline $\mathrm{P} 3 / \mathrm{P} 1$ & 2.5 & 13.9 & $\mathrm{~N} / \mathrm{A}$ & $\mathrm{N} / \mathrm{A}$ \\
\hline $\mathrm{P} 1 / \mathrm{N} 0$ & 2.0 & 5.5 & $\mathrm{~N} / \mathrm{A}$ & $\mathrm{N} / \mathrm{A}$ \\
\hline $\mathrm{P} 3 / \mathrm{N} 2$ & 1.5 & 5.5 & $\mathrm{~N} / \mathrm{A}$ & $\mathrm{N} / \mathrm{A}$ \\
\hline $\mathrm{N} 1 / \mathrm{N} 3$ & $\mathrm{~N} / \mathrm{A}$ & $\mathrm{N} / \mathrm{A}$ & 6.3 & 2.5 \\
\hline $\mathrm{P} 0 / \mathrm{P} 2$ & $\mathrm{~N} / \mathrm{A}$ & $\mathrm{N} / \mathrm{A}$ & 13.9 & 2.5 \\
\hline $\mathrm{N} 1 / \mathrm{P} 0$ & $\mathrm{~N} / \mathrm{A}$ & $\mathrm{N} / \mathrm{A}$ & 4.4 & 1.7 \\
\hline $\mathrm{P} 2 / \mathrm{N} 3$ & $\mathrm{~N} / \mathrm{A}$ & $\mathrm{N} / \mathrm{A}$ & 3.2 & 1.8 \\
\hline $\mathrm{N} 3 / \mathrm{N} 1$ & $\mathrm{~N} / \mathrm{A}$ & $\mathrm{N} / \mathrm{A}$ & 2.5 & 6.3 \\
\hline $\mathrm{P} 2 / \mathrm{P} 0$ & $\mathrm{~N} / \mathrm{A}$ & $\mathrm{N} / \mathrm{A}$ & 2.5 & 13.9 \\
\hline $\mathrm{P} 0 / \mathrm{N} 1$ & $\mathrm{~N} / \mathrm{A}$ & $\mathrm{N} / \mathrm{A}$ & 2.0 & 5.5 \\
\hline $\mathrm{N} 3 / \mathrm{P} 2$ & $\mathrm{~N} / \mathrm{A}$ & $\mathrm{N} / \mathrm{A}$ & 1.5 & 5.5 \\
\hline
\end{tabular}

manner. The data points represent the cross sections measured at regular intervals over 180 degrees of roll, at tilt angles greater than 40 degrees. For tilt angles less than forty degrees, the cross sections were vanishingly small.

It is worthwhile to note that the concept of effective LET itself is not valid in this case as the device SEU cross section is not uniquely defined at each tilt angle and primary species LET. In this case, there exists a span of two to four orders of magnitude in the measured cross section for equal values of effective LET over the range of roll angles. The broad range of upset cross sections and the strong roll dependence on cross section are not consistent with the assumptions of the traditional rectangular parallelepiped (RPP) model. As such, further analysis is required to identify the upset mechanisms and construct a model that accounts for the experimental observations.

\section{SEU ANALYSIS}

\section{A. SPICE Simulations}

SPICE analysis on the latch was performed for the purpose of identifying and quantifying the sensitive node or nodes and their respective critical charges. Single event charge collection was approximated using a double exponential current source. The peak amplitudes of the current sources were limited so that the potential of the evaluated node or nodes did not exceed the supply or ground rail. Charge was sunk or sourced from the drain of the off-state NMOS and PMOS transistors, respectively. The critical charge $\left(\mathrm{Q}_{\text {crit }}\right)$ was defined as the minimum charge or combination of charges required to upset the circuit and was calculated by the current source or sources over time.

The circuit was found to be immune to upset when only a single node of the device was struck. However, a number of twonode combinations were found to produce an error. Sensitive pairs depended on the state of the device and the critical charge was not the same for different pairs. This is illustrated in Table I, where for any struck transistor, $\operatorname{Tx}(\mathrm{A})$, the quantity of charge 
required on $\operatorname{Tx}(\mathrm{B})$ to cause an upset was less. Furthermore, the $\operatorname{Tx}(\mathrm{A})$ and $\mathrm{Tx}(\mathrm{B}) \mathrm{Q}_{\text {crit }}$ values did not commute for the N-P pairs (e.g., N0/P1 vs. P1/N0).

\section{B. TCAD Simulations}

While SPICE analysis can be used to demonstrate the circuit level effects of charge collection on single and multiple nodes, it does not capture the physical processes that must occur to result in the collected charge. To validate the proposed SEU mechanisms, two sets of three-dimensional mixed-mode TCAD simulations were performed to investigate simultaneous charge collection on the N0/N2 and the N0/P1 transistor pairs.

In this work we have used Davinci and Synopsys Dessis, commercial three-dimensional mixed-level device simulators. Doping profiles were derived from spreading resistance measurements of the process technology. Physical models used in the simulation included carrier concentration-dependent minority carrier lifetimes, Auger recombination, and doping dependent, electric field dependent, and carrier-carrier scattering mobility models. Ion strikes were modeled using a constant LET vs. depth and a Gaussian radial profile with a characteristic length of $0.1 \mu \mathrm{m}$. An iterative process of varying the incident LET was used to determine the threshold for which upset occurred. Two possible upset mechanisms are considered in the following sections.

1) N-N Simultaneous Charge Collection: The latch design was intended to provide sufficient separation between the N-N sensitive pairs to eliminate single particle charge-sharing mechanisms. However, the possibility of a particle at a high tilt angle traversing both transistors could not be eliminated.

Both transistors comprising the SEU-sensitive pair were simulated in three dimensions, while the rest of the latch (minus access transistors) was modeled with compact circuit models. The separation of the sensitive pair was based on the physical design layout. The single event strike was simulated at a tilt angle of 80 degrees, entering near the drain diffusion of $\mathrm{N} 0$ and passing beneath N2 (Fig. 4). Particle LETs between $5-10 \mathrm{MeVcm}^{2} / \mathrm{mg}$ were found to be sufficient to upset the latch.

2) N-P Upset Mechanism: The possibility of inducing a SEU by simultaneously depressing the NO drain and elevating the P1 drain nodes from a single strike was examined. The N0-P1 sensitive node pair was simulated as a TCAD structure with appropriately distanced well taps and the remaining portion of the circuit solved as SPICE elements.

The results of TCAD simulation (Fig. 5) show that a single particle corresponding to the $75^{\circ}$ tilt, $150^{\circ}$ roll heavy ion irradiation resulted in direct charge collection by the NMOS transistors with parasitic bipolar conduction in the off-state PMOS transistors due to n-well potential depression similar to that reported by Olson [11]. The minimum LET particle found to cause an upset was approximately $2.5 \mathrm{MeVcm}^{2} / \mathrm{mg}$.

\section{MRED Model AND CALIBRATION}

MRED is a Monte-Carlo simulation tool based upon the Geant 4 class libraries, which comprise computational physics models for the transport of radiation through matter [3]. The

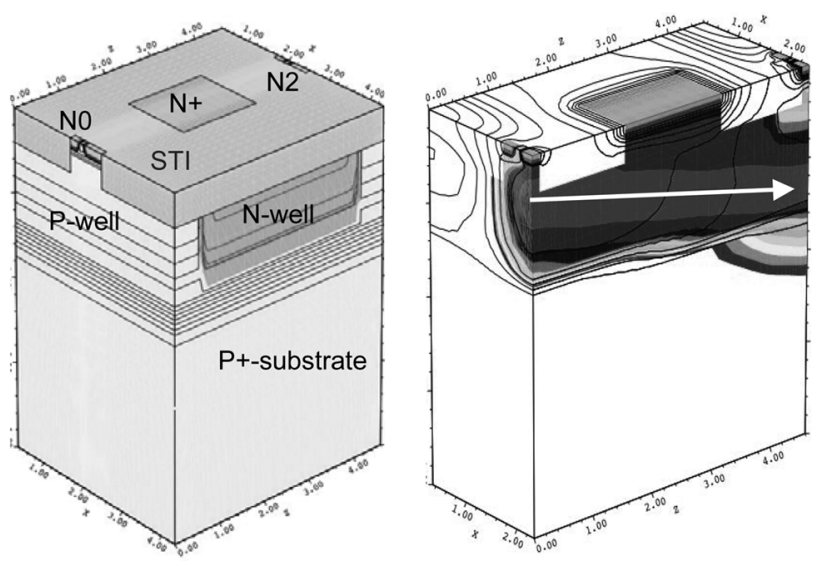

Fig. 4. Depiction of the TCAD model containing the N0 and N2 transistors separated by the n-well (left). The single event strike passes immediately beneath the drain of N0 in the direction of N2. Sufficient charge can diffuse from the track to both N0 and N2 to cause upset (right).
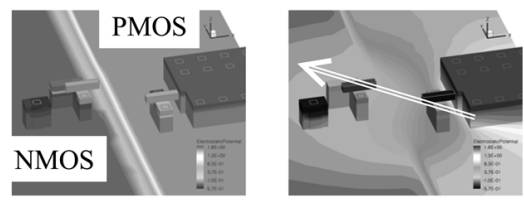

Fig. 5. TCAD simulation showing electrostatic potential in the vicinity of the N0-P1 transistor pair before a heavy ion strike (left) and the N-well potential collapse after the strike (right). The $\mathrm{N}$-well potential collapse resulted in parasitic bipolar conduction in the PMOS device.

application of MRED to modeling heavy ion SEU cross sections has been previously demonstrated [6], [12] for single and multiple bit upsets in static random access memories (SRAM).

The simulation flow for using MRED in modeling SEU cross sections and performing SEU rate calculations involves defining the following:

1) Target object: A spatial and compositionally accurate solid model of the irradiated device or devices through which the radiation is transported and in which energy deposition is monitored.

2) Sensitive volumes: Defines the location, size, and collection efficiency parameters of the sensitive volume set for each transistor. These are placed at user-defined locations within the target object.

3) Relationship between deposited energy and device upset: A definition of critical charges for each transistor, transistor pair, and any coincident requirements that cause a SEU such as those described in Table I.

4) The radiation environment: Defines the specifics of the heavy ion beam or the natural radiation environment. This includes the appropriate particle atomic number, mass, and energy as well as its trajectory.

\section{A. Solid Model Construction}

A solid model of the latch was generated using PROCEM, a module contained in the Synopsys Devise software. Each process layer (polysilicon, metal-1, metal-2, etc.) was derived from the layout files and SEM cross sections (Fig. 6).

MRED contains algorithms for replicating basic CAD objects. The mechanism reduces the memory requirements of the 


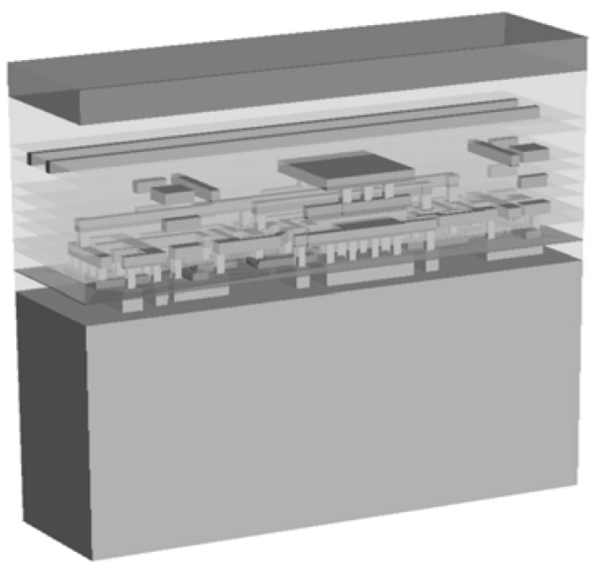

Fig. 6. Solid model of a single latch. The oxide and interlayer-dielectric material has been made transparent to illustrate the metallization layers.

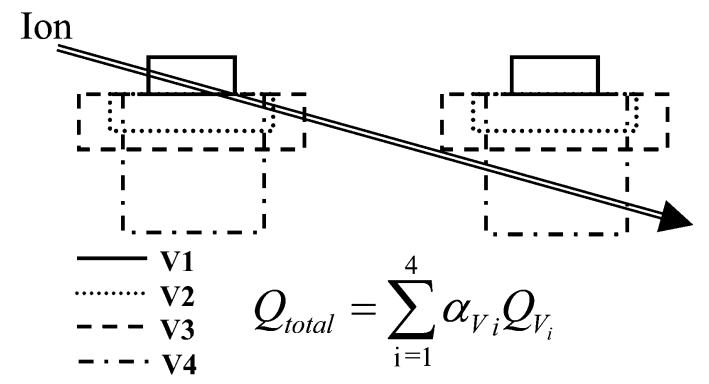

Fig. 7. Conceptual 2-dimensional cross sectional drawing of two sensitive volume sets, each of which defines an individual transistor. $Q_{\text {total }}$ is the net collected charge at the transistor node as a function of the charge generated in each sub-volume $\left(\mathrm{Q}_{\mathrm{Vi}}\right)$. The summation is performed separately for each individual transistor. The efficiency, $\alpha$, is an adjustable parameter and is determined during calibration to experimental data as well as from TCAD simulations.

simulation and is useful if one wishes to simulate a large array of identical structures. For this simulation, the solid model was replicated into a $5 \times 5$ bit array for the purpose of reducing possible edge effects as well as allowing the evaluation of cross sections on the programmed state of the device (e.g., all 1s, all 0s, checkerboards).

\section{B. Sensitive Volumes}

Single or multiple sensitive volumes can be defined within MRED. Furthermore, multiple sensitive volumes can be grouped together in such a way as to define the collected charge for a single transistor. The multiple sensitive volume concept has been previously presented in [6], [12] for MRED and in [13] for chord-length based error rate calculations.

The multiple sensitive volume concept is illustrated in Fig. 7 where four volumes are used to describe a single transistor. The total collected charge for either of the transistors is calculated as the weighted sum of the charge generated in each volume. Generally speaking, the highest collection efficiency parameter, $\alpha$, is associated with the volume containing the transistor drain node. Smaller values of $\alpha$ are selected for charge collection from strikes distant from the drain node or from deep within the substrate.

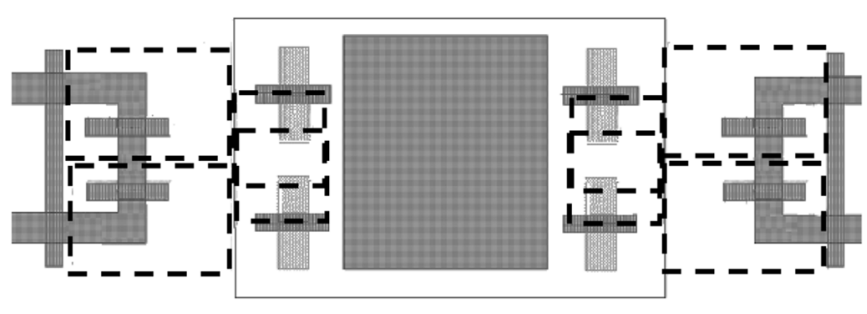

Fig. 8. Final placement of the sensitive volume set for each transistor with respect to the circuit layout. The dashed lines represent the outer boundary of the sensitive volume set for a transistor. Note that the PMOS sensitive volumes overlap.

The geometry and efficiency of the sensitive volumes are largely determined by physical boundaries within the device layout, such as well boundaries or adjacent transistors. The optimal depth of the volumes corresponds to junction depths such as the n-well (PMOS) or the epitaxial peak implant depth (NMOS). These values, including the final placement (Fig. 8) and charge collection efficiency parameters, are adjusted to produce good agreement with the experimental data.

\section{MRED SEU Conditions}

The circuit operation and SEU mechanisms of the hardened latch require that a minimum of two critical charge conditions must be satisfied to upset the circuit, where a total of four possible transistor combinations have 8 critical charge conditions for each logic state. For example, if a latch was programmed in a data state of ' 1 ', the simulator determined the collected charge of N0, N2, P1, and P3 following a radiation event. If any of the eight relevant combinations (Table I) of critical charges were satisfied, an upset was counted. This process was repeated 25 times per radiation event for each bit with the $5 \times 5$ array programmed in a checkerboard fashion.

\section{MRED Model Calibration}

Calibration of the sensitive volume parameter set (dimensions, placement, efficiencies) was achieved by repeated comparison of simulation results to the experimental data. The complete simulation set involved calculating the cross section for approximately 100 different heavy ion beam conditions (roll, tilt, and ion species).

Figs. 9-12 contain examples of the level of simulation fidelity achieved over a range of species, roll and tilt angles. The remainder of the experimental data and simulation results are shown in the Appendix . Rapid calibration through multiple simulations over all beam conditions was made possible by the use of the Vanderbilt University Advanced Computing Center for Research \& Education (ACCRE) VAMPIRE computing cluster [14].

The maximum NMOS and PMOS charge collection depths were determined to be 1.95 and $0.40 \mu \mathrm{m}$, respectively. The shallow tilt-angle cross sections were largely dominated by the N-P upset mechanism (close proximity, shallow collection depth) while at grazing incidence, the N-N mechanism was the most probable (large separation distance, deep charge collection depth). 


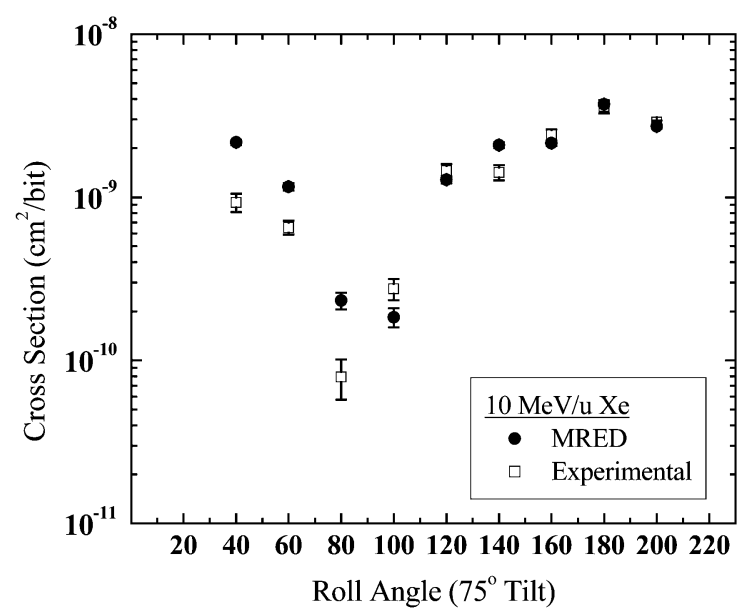

Fig. 9. SEU cross sections for the $10 \mathrm{MeV} / \mathrm{u}$ Xenon $\left(58.7 \mathrm{MeVcm}^{2} / \mathrm{mg}\right)$ beam at $75^{\circ}$ tilt as a function of roll angle. The highest SEU cross sections occur with the beam oriented along the long axis of the latch.

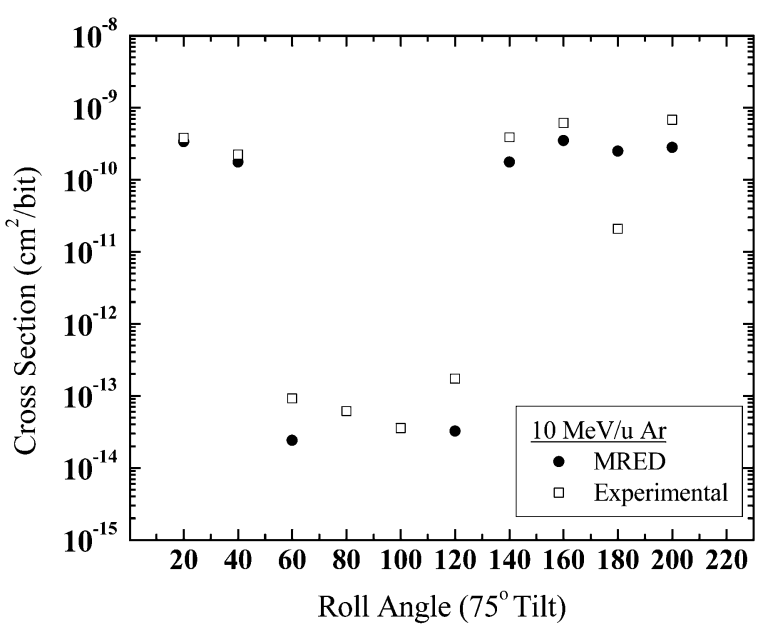

Fig. 10. SEU cross sections for the $10 \mathrm{MeV} / \mathrm{u} \operatorname{Argon}\left(9.7 \mathrm{MeVcm}^{2} / \mathrm{mg}\right)$ beam at $75^{\circ}$ tilt as a function of roll angle.

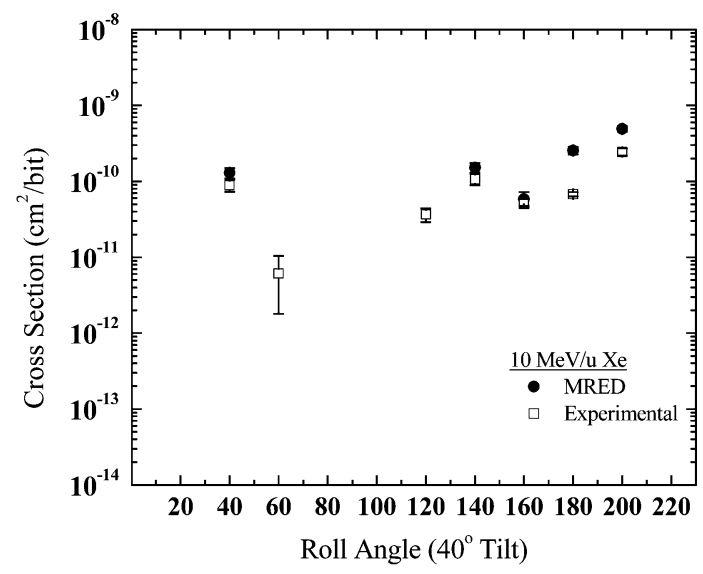

Fig. 11. SEU cross sections for the $10 \mathrm{MeV} / \mathrm{u}$ Xenon $\left(58.7 \mathrm{MeVcm}^{2} / \mathrm{mg}\right)$ beam at $40^{\circ}$ tilt as a function of roll angle.

At tilt angles at or below 40 degrees the experimental cross sections were the smallest. This is also the region of the largest discrepancy between the MRED model and experimental. An example is shown in Fig. 12 for Krypton at 40 degrees. The

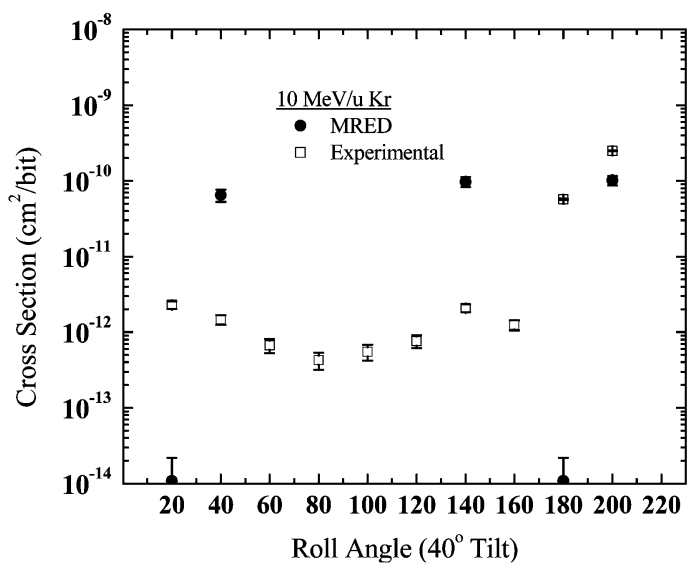

Fig. 12. SEU cross sections for the $10 \mathrm{MeV} / \mathrm{u}$ Krypton $\left(31.2 \mathrm{MeVcm}^{2} / \mathrm{mg}\right)$ beam at $40^{\circ}$ tilt as a function of roll angle. No SEU were recorded in MRED between 60 and 120 degrees.

is likely due to the geometrical approximation of the sensitive volumes as rectangular regions as well as the limitations associated with descritizing a spatially continuous physical process such as charge collection. Furthermore, the rate of decrease in cross section with decreasing tilt angle is at a maximum at $40 \mathrm{de}-$ grees, which adds to the difficulty in calibrating cross sections at the threshold. From an engineering perspective, over-predicting cross sections at a relatively small number of angles can be viewed as being conservative, but reasonable, for the final error rate prediction.

\section{RATE PREDICTION}

A soft error rate calculation for the calibrated model was performed using previously developed machinery available in MRED. The theoretical development and implementation of the rate prediction algorithms are discussed in [15]. In this study, GEO/interplanetary spectra during solar minimum for atomic numbers from 1 to 92 were selected and transported through 100 mils of aluminum using the CREME 96 TRANS algorithm [16]. Each spectrum was evaluated separately in MRED to determine the contribution of each atomic species to the on-orbit SER.

The true energy spectrum for each atomic species is sampled and transported through the solid model (Fig. 6) in a physical manner (e.g., screened coulomb scattering, hadronic interactions, etc....). No path-length-style calculations are associated with this type of simulation. Particle trajectories were randomly selected to mimic the isotropic nature of the natural space environment.

The result of the rate prediction, divided into the absolute contribution from each species, is shown in Fig. 13 for particles up to $\mathrm{Z}=65$. The combined SER rate is $2.3 \pm 0.8 \times 10^{-9} \mathrm{bit}^{-1}$ day $^{-1}$. The uncertainty is derived from counting statistics only and does not represent systematic errors in the model or spectra.

\section{DISCUSSION}

The process outlined in this work for calculating single event upset rates differs substantially from common analytical, path 


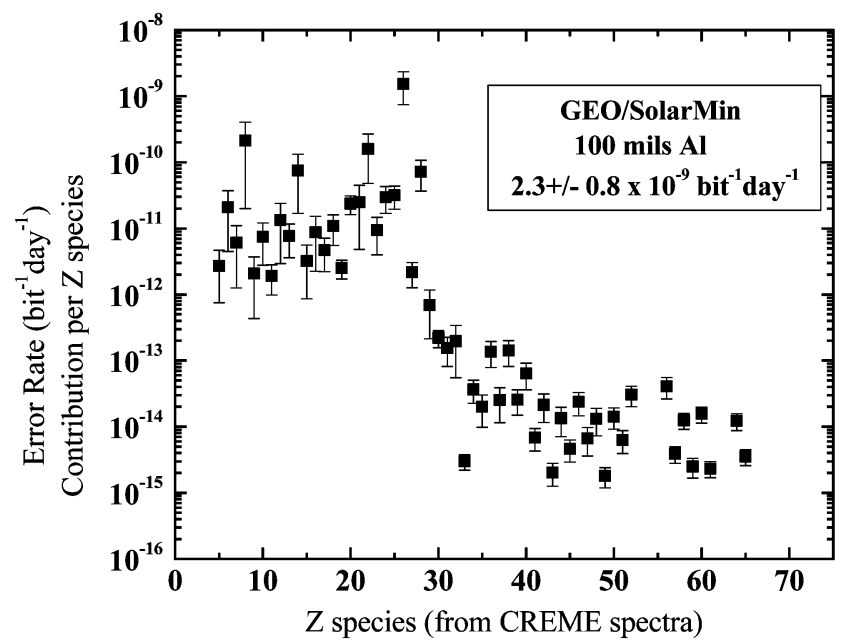

Fig. 13. Per-species SEU rate of the latch. The final calculated error rate was $2.3+/-0.8 \times 10^{-9}$ errors/bit-day.

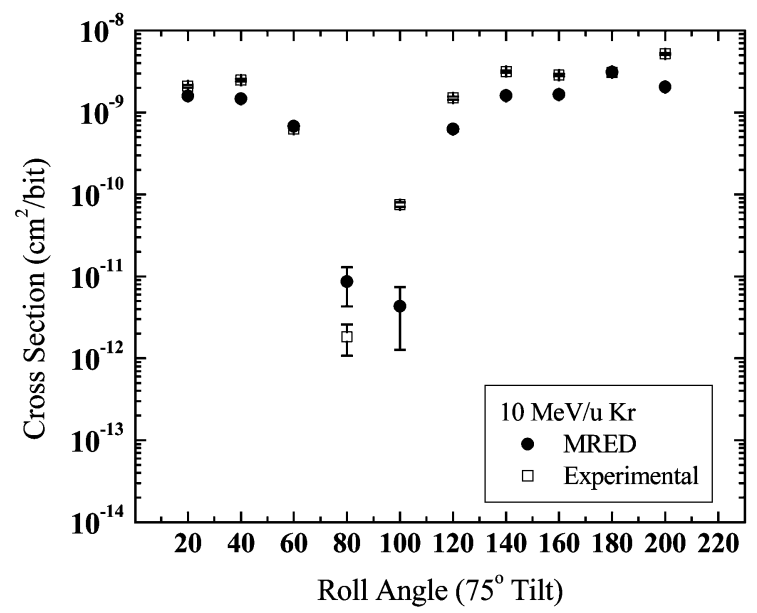

Fig. 14. SEU cross sections for the $10 \mathrm{MeV} / \mathrm{u}$ Krypton $\left(31.2 \mathrm{MeVcm}^{2} / \mathrm{mg}\right)$ beam at $75^{\circ}$ tilt as a function of roll angle.

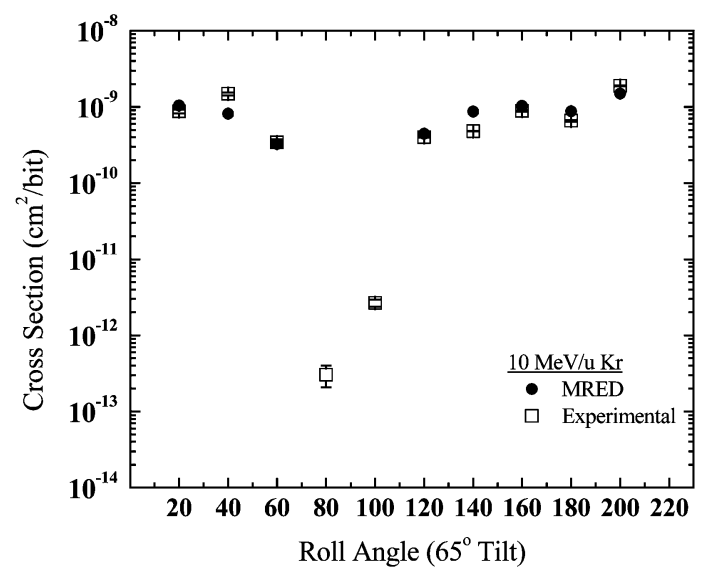

Fig. 15. SEU cross sections for the $10 \mathrm{MeV} / \mathrm{u}$ Krypton $\left(31.2 \mathrm{MeVcm}^{2} / \mathrm{mg}\right)$ beam at $65^{\circ}$ tilt as a function of roll angle.

length, SEU rate prediction methodologies. The technique allows the investigator to create a virtualization of the radiation environment, either those experienced in testing or in a natural space or terrestrial environment. Key aspects of the virtualization include:

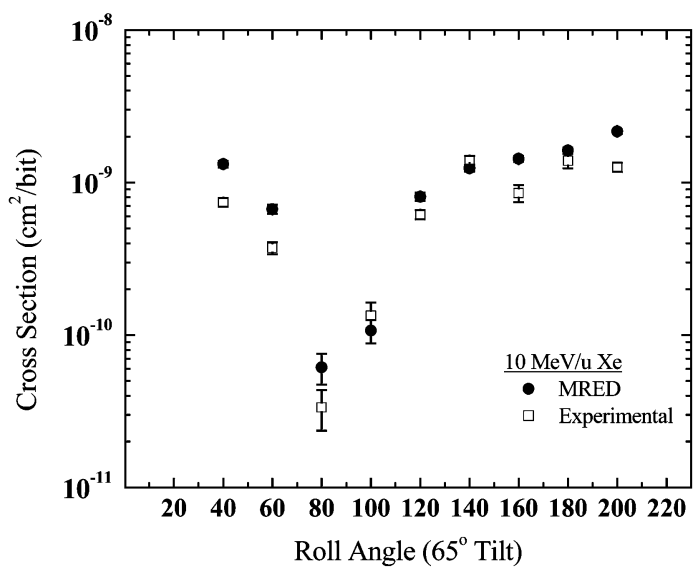

Fig. 16. SEU cross sections for the $10 \mathrm{MeV} / \mathrm{u}$ Xenon $\left(58.7 \mathrm{MeVcm}^{2} / \mathrm{mg}\right)$ beam at $65^{\circ}$ tilt as a function of roll angle.

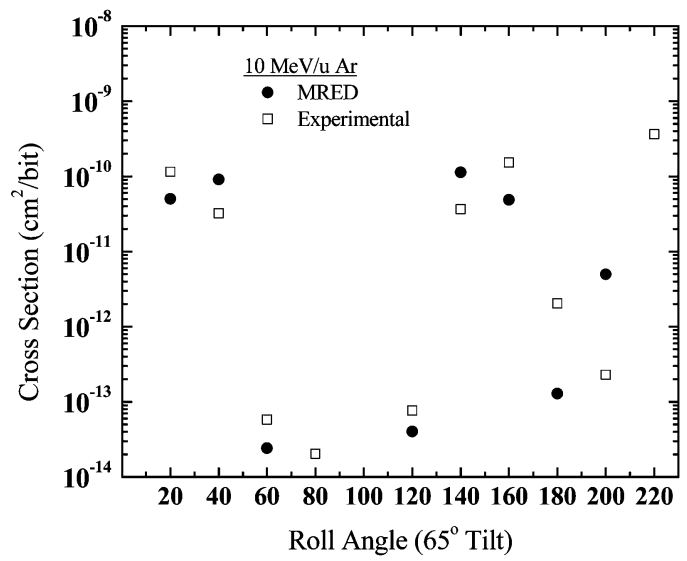

Fig. 17. SEU cross sections for the $10 \mathrm{MeV} / \mathrm{u} \operatorname{Argon}\left(9.7 \mathrm{MeV}-\mathrm{cm}^{2} / \mathrm{mg}\right)$ beam at $65^{\circ}$ tilt as a function of roll angle.

1) The transport of radiation is conducted using physical processes. Specifically, electronic and nuclear interactions between the incident particle and the target material are modeled and particles are tracked until they either stop in or exit the target object. As such, proton and heavy ion SEU can be evaluated simultaneously from the full radiation environment and when properly calibrated, separate DUT models for differing environments or particle beams are not required.

2) The description of the DUT is spatially and compositionally accurate. The stoichiometry and even isotopic composition of the target material can be specified to investigate potential impacts of existing and novel materials on single event response. The geometric accuracy provides a link between device layout, processing (e.g., metallization, interconnect density, passivation, interconnect metal type, etc.), and single event response.

3) The sensitive volume geometry and related parameters are correlated to transistor placement and calibrated against heavy ion or other (proton, neutron, etc.) test data. Additional simulation methods, such as the use of TCAD and SPICE, can assist in this process.

4) The mathematical relationship between energy deposition, charge collection, and valid SEU events is infinitely flexible. This allows one to develop a radiation response model (SEU, 
SET, etc.) with a level of precision that is adequate for the problem at hand (e.g., multi-volume charge sharing).

The level of complexity of the SEU response shown in Fig. 3 indicates that the concept of effective LET has no reasonable physical interpretation for devices whose upset response is driven by multiple node upset mechanisms. SEU cross sections cannot be viewed as single critical charge and simple path length calculation in a single rectangular volume. Rather, one must consider the charge characteristics of multiple transistors and the probabilities of coincident charge collection dominating the SEU response.

\section{CONCLUSION}

The latch design eliminates single node charge collection SEU vulnerabilities but is susceptible to multiple node charge collection processes. Specifically, charge collection from depths up to $2.0 \mu \mathrm{m}$ within the substrate in NMOS transistors and parasitic bipolar action in the PMOS transistors increase the probability of a single particle affecting multiple nodes. While physical separation of the NMOS transistors reduces the probability of N-N upsets, it is not completely eliminated for grazing incidence particles. Furthermore, improved means of isolating sensitive N-P pairs are required to improve SEU immunity.

The agreement between the model and the experimental data for the hardened latch not only supports the proposed mechanism and the simulation methodology, but also provides the maximum possible confidence in the final on-orbit soft error rate prediction.

In applications where extensive resources have been used to develop SEU immune or resistant storage elements, the on-orbit soft error rate is inherently a critical metric of the device's performance. However, as has been demonstrated, SEU hardened devices' cross sections and their error rates can be dominated by second order mechanisms that fall outside of the applicability of the classical single-volume RPP models. The flexibility of MRED (or potentially other suitably developed physics-based transport codes) allows one to overcome these limitations and helps to reduce the systematic uncertainty associated with the on-orbit error rate prediction process. Insight into the SEU mechanisms, both in the context of their relative contribution to heavy ion cross sections and the predicted SEU rate, provides valuable feedback to circuit and layout engineers to assist in improving the overall SEU immunity.

\section{APPENDIX}

Figs. 14, 15, 16, and 17 contain the remainder of the heavy ion data and the corresponding calibrated simulation results. The best visual agreement between experiment and simulation was achieved at the large tilt angles. As the tilt angle was reduced, experimentally measured SEU cross sections decreased substantially. The point at which the rate of decrease in cross section with respect to tilt angle was greatest represents that area with the greatest discrepancy between experiment and simulation.

\section{REFERENCES}

[1] J. D. Black, A. L. Sternberg, M. L. Alles, A. F. Witulski, B. L. Bhuva, L. W. Massengill, J. M. Benedetto, M. P. Baze, J. W. Wert, and M. G. Hubert, "HBD layout isolation techniques for multiple node charge collection mitigation," IEEE Trans. Nucl. Sci., vol. 52, no. 6, pp. 2536-2541, Dec. 2005.
[2] L. W. Connell and F. W. Sexton, "Further development of the heavy ion cross section for single event upset: Model (HICUP)," IEEE Trans. Nucl. Sci., vol. 42, no. 6, pp. 2026-2034, Dec. 1995.

[3] C. Inguimbert and S. Duzellier, "SEU rate calculation with Geant4 (comparison with CRÈME 86)," IEEE Trans. Nucl. Sci., vol. 51, no. 5, pp. 2805-2810, Oct. 2004.

[4] D. Lambert, J. Baggio, V. Ferlet-Cavrois, O. Flament, F. Saigne, B. Sagnes, N. Buard, and T. Carriere, "Neutron-induced SEU in bulk SRAMs in terrestrial environment: Simulations and experiments," IEEE Trans. Nucl. Sci., vol. 51, no. 6, pp. 3435-3441, Dec. 2004.

[5] C. L. Howe, R. A. Weller, R. A. Reed, M. H. Mendenhall, R. D. Schrimpf, K. M. Warren, D. R. Ball, L. W. Massengill, K. A. LaBel, J. W. Howard, and N. F. Haddad, "Role of heavy-ion nuclear reactions in determining on-orbit single event error rates," IEEE Trans. Nucl. Sci., vol. 52, no. 6, pp. 2182-2188, Dec. 2005.

[6] K. M. Warren, B. D. Sierawski, R. A. Weller, R. A. Reed, M. H. Mendenhall, J. A. Pellish, R. D. Schrimpf, L. W. Massengill, M. E. Porter, and J. W. Wilkinson, "Predicting thermal neutron-induced soft errors in static memories using TCAD and physics-based monte carlo simulation tools," IEEE Electron Device Lett., vol. 28, no. 2, pp. 180-182, Feb. 2007.

[7] S. Agostinelli, J. Allison, K. Amako, J. Apostolakis, H. Araujo, P. Arce, M. Asai, D. Axen, S. Banerjee, G. Barrand, F. Behner, L. Bellagamba, J. Boudreau, L. Broglia, A. Brunengo, H. Burkhardt, S. Chauvie, J. Chuma, R. Chytracek, G. Cooperman, G. Cosmo, P. Degtyarenko, A. Dell'Acqua, G. Depaola, D. Dietrich, R. Enami, A. Feliciello, C. Ferguson, H. Fesefeldt, G. Folger, F. Foppiano, A. Forti, S. Garelli, S. Giani, R. Giannitrapani, D. Gibin, J. J. Gomez, C. Cadenas, I. Gonzalez, G. Gracia Abril, G. Greeniaus, W. Greiner, V. Grichine, A. Grossheim, S. Guatelli, P. Gumplinger, R. Hamatsu, K. Hashimoto, H. Hasui, A. Heikkinen, A. Howard, V. Ivanchenko, A. Johnson, F. W. Jones, J. Kallenbach, N. Kanaya, M. Kawabata, Y. Kawabata, M. Kawaguti, S. Kelner, P. Kent, A. Kimura, T. Kodama, R. Kokoulin, M. Kossov, H. Kurashige, E. Lamanna, T. Lampen, V. Lara, V. Lefebure, F. Lei, M. Liendl, W. Lockman, F. Longo, S. Magni, M. Maire, E. Medernach, K. Minamimoto, P. Mora de Freitas, Y. Morita, K. Murakami, M. Nagamatu, R. Nartallo, P. Nieminen, T. Nishimura, K. Ohtsubo, M. Okamura, S. O'Neale, Y. Oohata, K. Paech, J. Perl, A. Pfeiffer, M. G. Pia, F. Ranjard, A. Rybin, S. Sadilov, E. Di Salvo, G. Santin, T. Sasaki, N. Savvas, Y. Sawada, S. Scherer, S. Sei, V. Sirotenko, D. Smith, N. Starkov, H. Stoecker, J. Sulkimo, M. Takahata, S. Tanaka, E. Tcherniaev, E. Safai Tehrani, M. Tropeano, P. Truscott, H. Uno, L. Urban, P. Urban, M. Verderi, A. Walkden, W. Wander, H. Weber, J. P. Wellisch, T. Wenaus, D. C. Williams, D. Wright, T. Yamada, H. Yoshida, and D. Zschiesche, "Geant4-a simulation toolkit," Nucl. Instrum. Methods Phys. Res. A, vol. A506, p. 250, 2003.

[8] [Online]. Available: http://www.python.org

[9] T. Calin, M. Nicolaidis, and R. Velazco, "Upset hardened memory design for submicron CMOS technology," IEEE Trans. Nucl. Sci., vol. 43, no. 6, pp. 2874-2878, Dec. 1996.

[10] R. Velazco, T. Calin, M. Nicolaidis, S. C. Moss, S. D. LaLumonDiere, V. T. Tran, and R. Koga, "SEU-hardened storage cell validation using a pulsed laser," IEEE Trans. Nucl. Sci., vol. 43, no. 6, pp. 2843-2848, Dec. 1996.

[11] B. D. Olson, D. R. Ball, K. M. Warren, L. W. Massengill, N. F. Haddad, S. E. Doyle, and D. McMorrow, "Simultaneous single event charge sharing and parasitic bipolar conduction in a highly-scaled SRAM design," IEEE Trans. Nucl. Sci., vol. 52, no. 6, pp. 2132-2136, Dec. 2005.

[12] K. M. Warren, R. A. Weller, B. Sierawski, R. A. Reed, M. H. Mendenhall, R. D. Schrimpf, L. W. Massengill, M. Porter, J. Wilkinson, K. A. LaBel, and J. Adams, "Application of RADSAFE to model single event upset response of a 0.25 micron CMOS SRAM," IEEE Trans. Nucl. Sci., accepted for publication.

[13] D. E. Fulkerson, D. K. Nelson, and R. M. Carlson, "BOXES: An engineering methodology for calculating soft error rates in SOI integrated circuit," IEEE Trans. Nucl. Sci., vol. 53, no. 6, pp. 3329-3335, Dec. 2007.

[14] [Online]. Available: http://www.accre.vanderbilt.edu

[15] R. A. Reed, R. A. Weller, R. D. Schrimpf, M. H. Mendenhall, K. M. Warren, and L. W. Massengill, "Implications of nuclear reactions for single event effects test methods and analysis," IEEE Trans. Nucl. Sci., vol. 53, no. 6, pp. 3356-3362, Dec. 2007.

[16] A. J. Tylka, J. H. Adams, J. P. R. Boberg, B. Brownstein, W. F. Dietrich, E. O. Flueckiger, E. L. Petersen, M. A. Shea, D. F. Smart, and E. C. Smith, "CREME 96: A revision of the cosmic ray effects on microelectronics code," IEEE Trans. Nucl. Sci., vol. 44, no. 6, pp. 2150-2160, Dec. 1997. 